\title{
A cross-sectional profile and outcome assessment of adult patients triaged away from Steve Biko Academic Hospital emergency unit
}

\author{
Andreas Engelbrecht ${ }^{\mathrm{a} *}$, FG du Toit ${ }^{\mathrm{b}}$ and MM Geyser \\ aFaculty of Health Sciences, Department of Family Medicine, Division of Emergency Medicine, Steve Biko Academic Hospital, Pretoria, \\ South Africa \\ ${ }^{b}$ Emergency Department, Kloof Hosptital, Pretoria, South Africa \\ 'Faculty of Health Sciences, Department of Family Medicine, University of Pretoria, and Head Emergency Unit, Kalafong Hospital, Pretoria, \\ South Africa \\ ${ }^{*}$ Corresponding author, email:engelbrechtandreas@gmail.com
}

\begin{abstract}
Background: Overcrowding is a global problem in emergency medicine. This study examined an approach to this problem at a central hospital.

Methods: A prospective observational study was done to provide a cross-sectional profile of patients triaged away from the emergency unit (EU) and to evaluate their outcome by telephonic survey.

Results: 549 patients were triaged away during the study period. There was no significant difference in the number of male and female patients in the sample. Female patients were significantly younger than males $(p=0.0399)$. The most common complaint was abdominal pain followed by extremity complaints. Females complained more of abdominal pains (OR 1.87, 95\% $\mathrm{Cl}[1.13-3.12] ; p$ 0.0094), and males had more extremity complaints (OR 2.42, 95\% Cl [1.45-4.09]; $p=0.0003$ ). Only 42 patients were available for telephonic follow-up; $66 \%$ of them received care on the same day at another treatment facility. No patients who were available for follow-up had died due to their presenting complaint or needed to be referred back.

Conclusions: The typical patient triaged away was a 40-year-old female from the hospital's referral area with abdominal pain. This study indicated that the method of triage may be safe to determine which patients can be diverted from a central hospital to a lower level of care. There were various limitations to this study; hence, the findings of this study should be interpreted with caution.
\end{abstract}

Keywords: emergency unit (EU), Medical Early Warning Score (MEWS), South African Triage Scale (SATS), triage

\section{Background}

Overcrowding in emergency units is a global and common phenomenon. Asplin et al. ${ }^{1}$ developed a conceptual model that partitions emergency unit crowding into three interdependent components: input, throughput and output. Different factors in each of these components have been highlighted in studies as factors contributing to crowding. ${ }^{2-4} \mathrm{~A}$ study by $\mathrm{McCabe}^{5}$ found that access block is the most important factor leading to crowding in the North American setting.

Inappropriate use of the emergency unit by non-critical patients was not found to be a major cause of crowding in some studies, but this was not tested in South Africa. ${ }^{1,6}$ A study done at New Somerset Hospital in Cape Town concluded that many low-priority patients are being seen in the emergency unit and should be managed by primary health care level staff. ${ }^{7}$ Other studies have also shown that emergency units often treat patients who can be treated at primary care centres..$^{5,8,9}$

Based on the above it would appear that long waiting times and expenditure at emergency units in South Africa could probably be reduced if non-critical patients presenting to an emergency unit could be referred to primary health care facilities.

Proven guidelines need to be in place to ensure that this denial of care in the emergency unit is not detrimental to the patient. Such guidelines currently do not exist in South Africa. Previous investigators have looked at ways to identify non-urgent patients in the emergency unit.
Versedi examined the use of the Canadian Triage Scale (CTS) as a tool to determine which patients can be sent away from the emergency unit. ${ }^{10}$ The results of this study showed that based on the CTS alone up to $17 \%$ of patients who needed emergency care would have been referred away. The study further determined that low-priority patients did not significantly contribute to emergency unit overcrowding. The author concluded that using the CTS as a tool to triage patients away is measurably unsafe and does not contribute to reducing emergency unit overcrowding.

Derlet et al. ${ }^{11}$ identified a subset of patients that can be safely triaged out of the emergency unit without any adverse outcomes based on certain criteria.

The criteria used in this study included vital signs, certain high-risk indications (severe pain, chest or abdominal pain, inability to walk, age $<16$, high-risk condition), a focused clinical examination based on complaint and a list of non-emergency chief complaints. These criteria are very similar to criteria used in the South African Triage Scale (SATS).

The SATS uses a modified version of the Medical Early Warning Score (MEWS) as basis for triage. Previous studies have shown that MEWS scores of 5 or more were associated with increased risk of death and ICU admission. ${ }^{12}$ A MEWS score of 5 or more would correlate with a SATS orange category (available from http://emssa.org.za/sats/). It follows that these patients could not be safely triaged out of an emergency centre. 
Patients with a SATS category of yellow or green can possibly be triaged to other treatment centres but no studies exist that show a correlation between these categories and disposition or outcome.

A recent study assessed the need for hospital admission by the Cape triage discriminator presentations. ${ }^{13}$ These discriminators also form part of the South African Triage Score. In this study the discriminators were inferior to the Simple Clinical Score (SCS) to predict outcome. The discriminator list classified nearly all patients in the high-risk group based on the SCS as urgent or very urgent (orange or red). Of 60 patients classified as low risk based on the SCS only 13 had delayed classifications based on the discriminators (green or yellow). The study concluded that the discriminator list predict soutcome poorly.

This study was undertaken to test the current practice at Steve Biko Academic Hospital, which is similar in some regards to procedures used during the study of Derlet et al. ${ }^{11}$

\section{Methods}

This prospective observational descriptive study was performed at Steve Biko Academic Hospital, a central-level state hospital in Gauteng, South Africa. A consecutive sample of adult patients presenting to the emergency unit and triaged away to another treatment facility during a four-week period from July 4, 2011 to July 31, 2011 was included in this study. During this period 549 adult patients were selected and included for analysis.

Patients presenting to the emergency department and not in need of immediate care were seen by a triage nurse who obtained a history from the patient. Vital signs were recorded and the patients were scored using the South African Triage Score. All patients were then assessed by a doctor on duty to decide whether the patient still needed to be seen in the emergency unit or referred to another treatment facility. The general recommendation in this unit is that only patients in the green and yellow categories should be considered for such a triage referral.

Patients triaged away from the emergency unit who provided consent and a contact telephone number were contacted after at least three days to determine the outcome of their condition.

Adverse outcomes were defined as:

- death of the patient within $72 \mathrm{~h}$ due to the same condition;

- admission of the patient to a same-level treatment facility;

- referral to a specialist for the same condition.

Table 1: Patient demographics $(n=549)$

\begin{tabular}{lcc}
\hline & Male & Female \\
\hline$n$ & 254 & 295 \\
Age: mean (SD) & $43.34(17.82)$ & $40.25(17.29)$ \\
Age: median (IQR) & $41(28-54)$ & $36(26-52)$ \\
\hline
\end{tabular}

Note: Overall mean age is 41.68 years (SD 17.59), median 39 years (IQR 26-53).

Table 2: Living area $(\boldsymbol{n}=549)$

\begin{tabular}{lc}
\hline Inside referral area & $415(75 \%)$ \\
Outside referral area & $125(23 \%)$ \\
No living area supplied & $9(2 \%)$ \\
\hline
\end{tabular}

Patients were also asked if they did seek further medical care on the same day after being referred away from the emergency unit. All patients deflected away from the emergency unit received a triage note with a management plan including which alternative health care facility to attend. Hence it was expected that most of them would attend an alternative facility.

Clarification of the selection of adverse outcomes: A system where a patient is denied emergency care and dies within three days due to the presenting complaint would be undesirable. The system used to decide which patients to send away should be sensitive enough that no patients with life-threatening conditions are triaged away. However, it should be noted that in the setting where the study was conducted, admission of terminally ill patients to the central hospital with limited beds would be inappropriate and referral to more appropriate facilities should be made. In the setting of this study a district hospital is available for such patients, located within walking distance of the central hospital.

The triage system should thus ideally identify patients who need the expertise of a central hospital and not necessarily a lower level hospital. Referring patients away only for them to be referred back on the same day for central hospital care would be undesirable.

Data from the triage forms and answers from the telephonic follow up were entered into an electronic database (Access 2007) and then exported to Excel 2010 and PSPP for further statistical analysis.

\section{Ethics}

The study was approved by the Ethics Committee of the University of Pretoria. During the period of the study all patients presenting to the emergency unit for triage were given an option to participate in the study. Those patients who chose to participate completed a patient information leaflet and consent form before being included in the study. Patients who chose not to participate were not penalised in any way. In order to maintain patient confidentiality no identifying personal information was recorded on the data-capturing sheet.

\section{Results}

A total of 549 adult patients were triaged away to other treatment facilities during the study period. Table 1 shows the basic demographics of this group. There was no significant difference in the number of males and females being triaged away. Female patients were significantly younger than males $(p=0.039$ ). The graph showing the age of patients triaged away from the unit was positively skewed. Unfortunately data are not available to compare whether this reflects the normal distribution of patients presenting to the emergency unit or whether a higher percentage of younger people are triaged away.

The areas in which the patients live or work were compared with the guidelines for referral areas in Gauteng as issued by the Department of Health. Results are shown in Table 2. Most patients came from within the referral area of the hospital.

Figure 1 shows the distribution of patients during the study period at different times of the day. The graph of triage times showed a bimodal distribution with most patients triaged away between 8 am and $11 \mathrm{am}$, and $7 \mathrm{pm}$ and $8 \mathrm{pm}$. This follows the perceived normal pattern of busy times in the emergency unit although no data exist to support this view. 


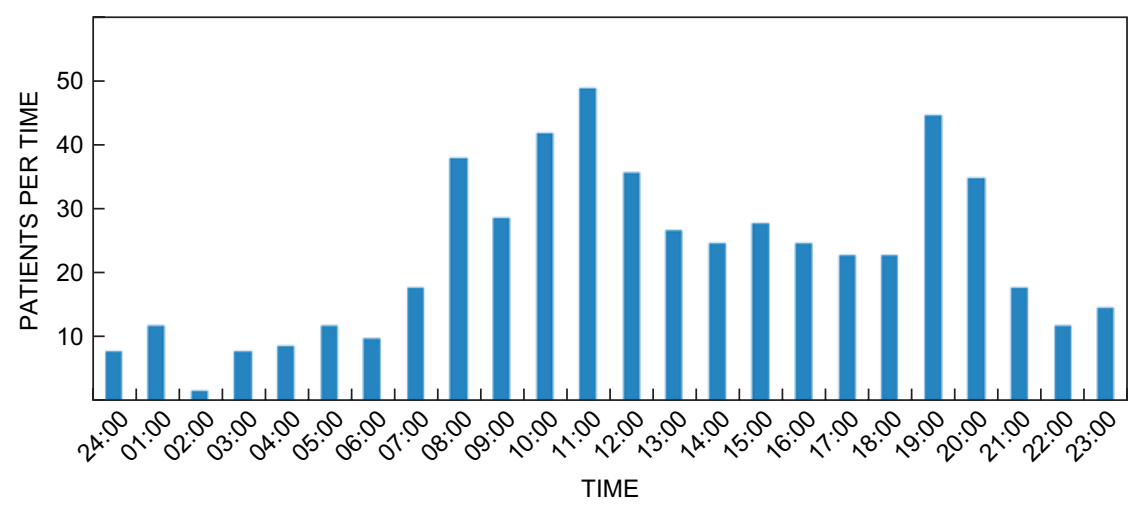

Figure 1: Patients per time

Figure 2 shows the number of patients triaged away per day during the study period. There were four days on which significantly more patients were triaged away than normally. This was on July 9, 11, 22 and 24. On four days significantly fewer patients than normal were triaged away. This was on July 14, 15, 19 and 26. It is unclear why there was a deviation from the mean on these days.

Figure 3 shows the number of patients seen on each day of the week. An average of 19 people per day were triaged away to other treatment facilities. No significant deviation from the mean was seen for specific days of the week.

Table 3 gives the breakdown of triage categories. Most of the patients in the study had a South African Triage Scale (available from http://emssa.org.za/sats/) category of green. The second most common category was patients to whom no triage category was assigned, then those with a yellow triage score, then orange and lastly red.

The main complaints of patients were reduced to common categories. The most common complaint was abdominal pain followed by extremity complaints. Results are given in Table 4 . Females complained more of abdominal pains (OR 1.87, 95\% Cl 1.13-3. 12) and males had more extremity complaints (OR 2042, $95 \% \mathrm{Cl} 1045-4.09) ; p=0.0003$.

Only $42(7.65 \%)$ patients completed the consent form with a contact number for follow-up. These patients were contacted by telephone and asked about their further medical care. The questions that were asked are listed in Table 5.

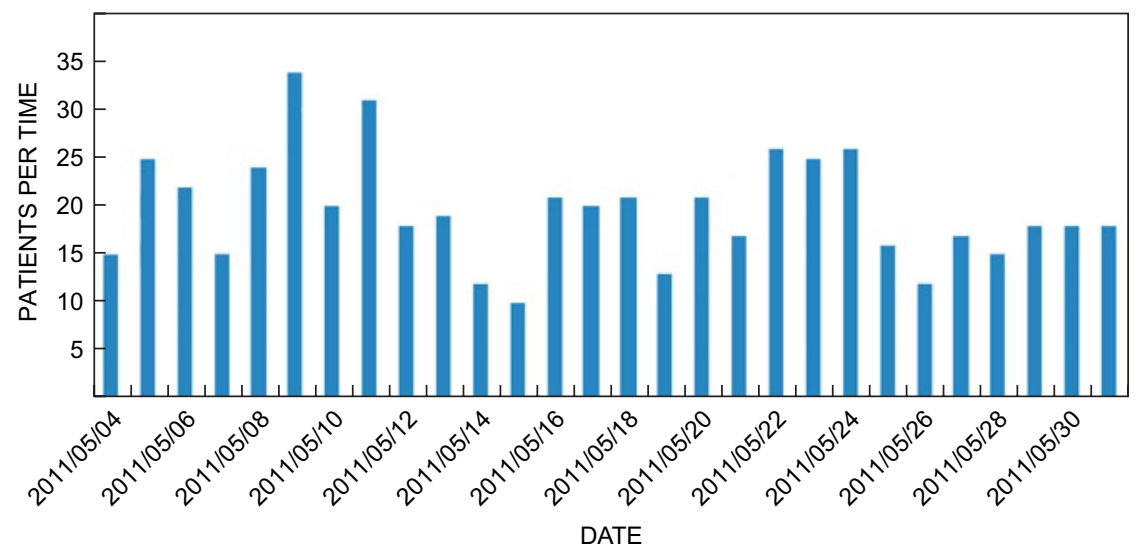

Figure 2: Total patients per day

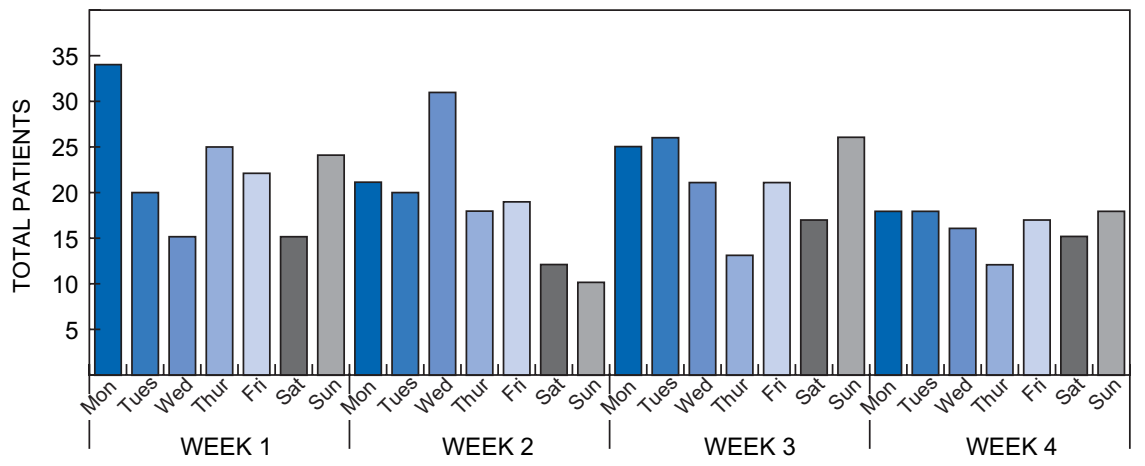

Figure 3: Total patients seen on days of the week 
Table 3: Triage categories

\begin{tabular}{lcc}
\hline & Total & $\%$ \\
\hline Green & 263 & 47.91 \\
Yellow & 67 & 12.20 \\
Orange & 23 & 4.19 \\
Red & 2 & 0.36 \\
Blank & 194 & 35.37 \\
Total & 549 & 100 \\
\hline
\end{tabular}

The outcome of the patients triaged away and available for follow-up is given in Table 6 . Of the 549 patients included in this study only 42 patients completed the consent form for telephonic follow-up and provided a telephone number. Of these patients only 31 completed the telephonic questions. Unfortunately some of the telephone numbers provided were incorrect, telephone numbers were for residences were patients did not permanently reside or there was no answer at the number despite several attempts. Only 22 of the 263 patients with a green triage category, none of the 67 patients with a yellow triage category, 1 of the 23 patients with an orange triage category, 1 of the 2 patients with a red triage category and 7 of the 194 patients without a triage category provided information on outcome. This low number of patients limits the conclusions that can be drawn from the outcome data.

\section{Discussion}

This study showed that the typical patient triaged away was a 40-year-old female, from within the hospital's referral area, with abdominal pain. Most patients referred to other facilities initially received a SATS triage category of green and sought further medical care on the same day at the site referred to. Female patients complaining of abdominal pain were the most common type of patient triaged away for alternative care. Conversely most male patients who were triaged away had minor extremity complaints. This difference may be due to the higher rate of trauma in male patients and the higher rate of pelvic problems in female patients.

No negative outcome to patients deflected from the emergency unit was detected in this study. None of the patients with a green or yellow category had an adverse outcome at the time of follow-up. This finding seems to support the current practice of selecting patients with these triage outcomes to be seen at other treatment facilities. It also lends support to the view that emergency unit crowding could be due to people using the emergency unit rather than more suitable primary care facilities as stated by Hodkinson et al. ${ }^{7}$ However, these conclusions must be viewed with caution based on the small number of patients available for follow-up.

A further problem was the significant number of forms that had no triage category assigned. The exact reason why this occurred

Table 4: Common presenting complaints

\begin{tabular}{|c|c|c|c|c|}
\hline & Female & Male & $n$ & $\%$ \\
\hline Abdominal pain & 59 & 30 & 89 & 16.21 \\
\hline Extremity complaints & 29 & 53 & 82 & $14.94 \%$ \\
\hline Chest pain & 28 & 17 & 45 & 8.2 \\
\hline Coughing & 8 & 21 & 29 & 5.28 \\
\hline Headache & 16 & 11 & 27 & 4.92 \\
\hline PV bleeding & 26 & 0 & 26 & 4.73 \\
\hline Back pain & 13 & 11 & 24 & 4.37 \\
\hline Difficulty in breathing & 16 & 8 & 24 & 4.37 \\
\hline Vomiting & 9 & 8 & 17 & 3.10 \\
\hline Others & & & & \\
\hline
\end{tabular}

Table 5: Telephone questions

Did you go to the referred hospital on the same day?

Did the you/the patient die from the presenting complaint within three days?

Were you admitted to another hospital within three days?

Were you seen by a specialist for your complaint?

Were you referred back to the same hospital for specialist care?

Table 6: Outcome of patients triaged away $(n=42)$

\begin{tabular}{|c|c|c|c|c|c|c|}
\hline & Green & Yellow & Orange & Red & None & Total \\
\hline Same day medical care & $22(52.38 \%)$ & 0 & $1(2.38 \%)$ & 0 & $6(12.77 \%)$ & $29(69.05 \%)$ \\
\hline Died & 0 & 0 & 0 & 0 & 0 & 0 \\
\hline Admission & 0 & 0 & 0 & $1(2.38 \%)$ & $1(2.38 \%)$ & $2(4.76 \%)$ \\
\hline Specialist & 0 & 0 & 0 & 0 & 0 & 0 \\
\hline Referral back to hospital on same day & 0 & 0 & 0 & 0 & 0 & 0 \\
\hline Total & 29 & 1 & 1 & 1 & 10 & 42 \\
\hline
\end{tabular}


was unclear. Many cases appeared to be in triage category green where the triage nurse may have considered it unnecessary to document the triage colour. It was further noted that the triage category was incorrectly assigned on many forms. For example, abdominal pain should push a patient in category green up to category yellow. Many patients with abdominal pain were still given a green triage category. Although most of these cases reflect an error in the triage category assignment process some may reflect a clinical judgement call. The double assessment system used at the hospital allows patients to be moved from a higher to a lower triage category based on the final medical assessment. This assessment follows the nursing assessment.

It was of concern that a number of patients with an orange and red triage category were referred to other treatment facilities. The current South African Triage Score recommends treatment within $10 \mathrm{~min}$ as a goal for patients with an orange triage category and immediate treatment for patients with a red category. Sending a patient to another treatment facility, even when close by, would in all cases result in delaying treatment by more than $10 \mathrm{~min}$. Some of the patients in the orange category and both the patients in the red category probably reflect clinical decision errors of the triage officers involved. These kinds of clinical decision errors are picked up by research and reflect the importance of audit and feedback to triage officers in order to limit mistakes. Although some of these patients may have had terminal conditions such as end-stage cancer or HIV disease and could thus have been appropriately sent to the nearby district hospital for end-of-life care it can still be argued that they should have been stabilised witdhin the emergency unit prior to being sent away.

In contrast to the study done by Diensburg-Stanwood et al. ${ }^{9}$ where only $40 \%$ of patients attended another treatment facility, almost $66 \%$ of patients in this study did seek medical treatment on the same day at another treatment facility. In the study by Darlet et al. ${ }^{11} 42 \%$ of patients received care on the same day. The observed difference could be due to the physical set-up at the site where the study was conducted where a district hospital emergency unit with $24 \mathrm{~h}$ service and a primary health care clinic are located adjacent to the study site and within walking distance. A further reason might be the type of patients triaged away. In the study done by Darlet et al. ${ }^{11}$ symptoms such as abdominal pain and chest pain excluded patients from being triaged away while many patients triaged away in this study had such complaints. Further reasons why such a high number of patients sought help on the same day may include the provision for a management plan on the triage form. The medical assessment and discussion may have motivated patients to seek help on the same day.

It would appear that overcrowding in this EU could be averted or improved if primary healthcare facilities were better used. Public awareness programmes on when to use the EU and when to use a primary healthcare facility are lacking and may improve public understanding of how to correctly access healthcare facilities and family medicine practices rather than the EU.

Previous studies have found that triaging patients away from the emergency unit does not reduce crowding. ${ }^{8,13}$ In this study an average of 19 patients per day were triaged away. This may have had a significant impact on the reduction of crowding in the emergency unit but no data are available to support this view. Further study into emergency unit length of stay and factors affecting crowding in this specific setting need to be done to confirm this. The high number of patients that were triaged away may reflect a failing primary health care system. The reasons why patients with primary health care problems present to a tertiary hospital needs to be explored with further research.

Only $7 \%$ of patients were available for telephonic follow-up. Other similar studies with telephonic follow-up resulted in follow-up rates of $37 \%$ and $40 \% .{ }^{11}$ The low rate of follow-up might be a result of patients misunderstanding the patient information leaflet and believing that providing a telephone number might result in them being sent to another treatment facility. In planning further research in this area it should be taken into account that telephonic follow-up of patients often results in suboptimal numbers. An alternative to this approach would be to conduct a larger study over a longer period of time to obtain a larger more representative sample of patients to assess.

\section{Limitations}

One of the major limitations in this study was the inability to obtain consent from more patients to provide follow up on their patient care.

A number of patients who were triaged away and gave consent to be contacted could not be reached. Some of these patients may have had an adverse event that could not be recorded.

Most of the patients in this study (who were triaged away) were in the green triage category. This category was determined by a nursing assessment using the SATS score followed by a medical assessment. It was noted that many of the patients had discriminators such as abdominal pain that should have pushed them into a higher triage category. Some of these cases may have been kept in the lower triage category due to clinical judgement but many probably indicate incorrect triage assignment. The effect of these cases of incorrect triage category assignment is uncertain. A significant number of patients also did not have a documented triage category assigned to them.

The findings of this study are further limited by the short period of data collection. There are only a number of cases known to the institution where the study was conducted of bad outcome after patients were triaged away over a number of years of this practice. A longer study period would be more likely to have included such an adverse outcome and allow examination of possible causes.

As this study looked only at those patients triaged away from the unit it is not known whether patterns observed in these patients mirror those of all patients seen in the unit. This study aimed only to describe the profile of patients triaged away and not the differences between those admitted and discharged.

Only adult patients were included in this study and thus no conclusions can be made about triaging children away from an emergency unit.

Data were only collected at a tertiary hospital where there is relatively easy access to a primary health care facility and district hospital. This'safety net' is not applicable in many other locations, thus limiting the generalisability of the findings of this study.

Data obtained from the triage forms were often incomplete or incorrect, limiting the conclusion that can be made from these data regarding the SATS as an aid in deciding on patient deposition. 


\section{Conclusions}

Based on the foregoing results and discussion the following conclusions are made:

- The profile of patients in this study indicates that the typical patient triaged away from this unit is a female in her mid-thirties to forties with abdominal pain. Males commonly had extremity injuries. These patients came from within the hospital's referral area and mostly presented during office hours or the early evening.

- While findings of this research seem to support the safety of the current practice at the site of the study, significant limitations such as loss to telephonic follow-up and incorrect assignment of triage category mandate that the results should be interpreted with caution. Further research with better follow-up is needed to confirm or reject this finding.

- The referral of patients with an orange or red triage category is ethically questionable and probably reflects clinical error in many cases. It also emphasises the importance of triage audit to pick up these cases in order to assist triage officers to recalibrate and improve their practice. Retraining of staff performing triage and frequent audit of triage statistics is recommended to improve the quality of SATS scoring and the data available for research.

\section{References}

1. Asplin BR, Magid DJ, Rhodes KV, et al. A conceptual model of emergency department crowding. Ann Emerg Med. 2003;42:173-80. http://dx.doi.org/10.1067/mem.2003.302

2. Thompson C, Hayhurst C, Boyle A. How have changes to out-of-hours primary care services since 2004 affected emergency department attendances at a UK District General Hospital? A longitudinal study. Emerg Med J.2010;27:22-5.http://dx.doi.org/10.1136/emj.2008.068817
3. Buffard MJ, Villa-Roel C, Bond K, et al. Tracking emergency department overcrowding in a tertiary care academic institution. Healthc Q. 2009;12(3):99-106.

4. Cook R, Thakore S, Morrison W, et al. To ED or not to ED: NHS 24 referrals to the emergency department. Emerg Med J. 2010;27:213-5. http://dx.doi.org/10.1136/emj.2008.064261

5. McCabe J. Emergency department overcrowding. Acad Med. 2001;76:672-4. http://dx.doi.org/10.1097/00001888-200107000-00005

6. Schull MJ, Kiss A, Szalai J-P. The effect of low-complexity patients on emergency department waiting times. Ann Emerg Med. 2007;49: 257-64. http://dx.doi.org/10.1016/j.annemergmed.2006.06.027

7. Hodkinson PW, Wallis LA. Cross-sectional survey of patients presenting to a South African urban emergency centre. Emerg Med J. 2009;26:635-40. http://dx.doi.org/10.1136/emj.2008.063362

8. Gentile S, Vignally $P$, Durand $A C$, et al. Nonurgent patients in the emergency department? A French formula to prevent misuse. BMC Health Serv Res. 2010;38:1-8.

9. Diesburg-Stanwood A, Scott J, Oman K, et al. Non-emergent ED patients referred to community resources after medical screening examination: characteristics, medical condition after 72 hours, and use of follow-up services. J EmergNurs. 2004;30:312-7.

10. Vertesi, L. Does the Canadian Emergency Department Triage and Acuity Scale identify non-urgent patients who can be triaged away from the emergency department? CJEM. 2004 Sept 5;6:337-42.

11. Derlet RW, Richards JR. Overcrowding in the nation's emergency departments: complex causes and disturbing effects. Ann Emerg Med. 2000;35:63-8. http://dx.doi.org/10.1016/S0196-0644(00)70105-3

12. Burch VC, Tarr G, Morroni C. Modified early warning score predicts the need for hospital admission and inhospital mortality. Emerg Med J. 2008;25:674-8. http://dx.doi.org/10.1136/emj.2007.057661

13. Emmanuel A, Ismail A, Kellet J. Assessing the need for hospital admission by the cape triage discriminator presentations and the simple clinical score. Emerg Med J. [Internet] 2010 [cited 2010 May 31]. Available from: http://emj.bmj.com/

Received: 12-10-2014 Accepted: 21-02-2015 